Essay

\title{
Evolutionary change: facultative virulence by brood parasites and tolerance and plastic resistance by hosts
}

\author{
Juan J. Soler ${ }^{a,}{ }^{*}$, Manuel Soler ${ }^{b}$ \\ a Departamento de Ecología Funcional y Evolutiva, Estación Experimental de Zonas Áridas (EEZA-CSIC), Almería, Spain \\ ${ }^{\mathrm{b}}$ Departamento de Zoología, Facultad de Ciencias, Universidad de Granada, Granada, Spain
}

\section{A R T I C L E I N F O}

\section{Article history:}

Received 10 October 2016

Initial acceptance 7 November 2016

Final acceptance 27 December 2016

MS. number: 16-00886R

\section{Keywords:}

antiparasitic defence

arms race

Clamator glandarius

coevolution

coevolutionary cycles

Cuculus canorus

diversification

parasite specialization
Coevolutionary theory predicts that parasitism selects for defensive traits in the hosts that counteract the negative effects of parasites. Such antagonistic interactions may continuously coevolve within cycles without end, or result in host specialization and speciation of parasites. Here, we argue that particularities of brood parasite-host systems explain whether the coevolutionary relationships result in parasite specialization and speciation. Highlighted particularities of the system are (1) virulence of the parasites, (2) the ability of parasites to alter host behaviour, (3) the relative importance of defensive tolerance and defensive resistance of hosts, and (4) phenotypic plasticity of parasite virulence and host resistance. Fixed virulence of parasites selects for fixed resistance of hosts and both enhance the process of specialization and speciation of parasites. In contrast, phenotypic plasticity in virulence of the parasites would select for tolerance and facultative resistance in their hosts. These host characteristics imply limited escalation in resistance defences and therefore would facilitate continuous coevolutionary cycles preventing parasite specialization. Thus, when studying the diversification of brood parasites within the avian phylogeny, considering these three factors would help us understand what drives their evolution. To illustrate the importance of virulence, phenotypic plasticity and defensive tolerance for the evolution of parasites, we compare evolutionary radiation experienced by the genus Clamator and the Tribe Cuculinii, which includes the genus Cuculus, and speculate whether particularities of brood parasitism by the great spotted cuckoo, Clamator glandarius, and the common cuckoo, Cuculus canorus, explain differences in evolutionary radiation experienced by these two groups of brood parasites.

๑ 2017 Published by Elsevier Ltd on behalf of The Association for the Study of Animal Behaviour.
Parasites obtain benefits from other individuals (hosts), thereby decreasing the hosts' fitness. Coevolutionary theory predicts that parasitism selects for defensive traits in the hosts that counteract the negative effects of this antagonistic relationship (Thompson, 1994). Such antagonistic interactions may continuously coevolve within cycles without end, or result in host specialization and speciation of parasites. Hosts evolve to resist and/or to tolerate parasite attacks, which would imply different evolutionary outcomes and affect the speed of evolutionary change (Svensson \& Råberg, 2010). While defensive resistance refers to characteristics that prevent or release hosts from parasitism, defensive tolerance applies to host characteristics that reduce the negative fitness effects of parasitism without affecting parasite fitness. Immune responses are examples of resistance defences because they eliminate

\footnotetext{
* Correspondence: M. Soler, Departamento de Zoología, Facultad de Ciencias, Universidad de Granada, E-18071 Granada, Spain.

E-mail address: msoler@ugr.es (J. J. Soler).
}

parasites from hosts, while red blood cell disorders that reduce the incidence of malarial parasites without affecting parasite loads is considered defensive tolerance (Råberg, Graham, \& Read, 2009). The relative importance of both defensive strategies and, therefore, characteristics of the evolutionary process depends on parasite virulence and other particularities of the host-parasite system (Medzhitov, Schneider, \& Soares, 2012; Moore, 2002; Svensson \& Råberg, 2010).

Virulence, defined as the strength of negative effects of parasitism, selects for strong defensive resistance in the parasites' hosts, which reduces parasite fitness and, among other counter-defences, may select for increased virulence in parasites (Schmid-Hempel, 2011). Thus, reciprocal evolutionary change in both the parasite and the host species triggering successive defences and counterdefences, which is known as a coevolutionary arms race (Dawkins \& Krebs, 1979), will be driven by the level of virulence of the parasite and the intensity of the evolved host defences via resistance. In contrast, reciprocal evolutionary changes would be expected to occur slowly, if at all, in host-parasite systems in which 
hosts mainly show defensive tolerance (Best, White, \& Boots, 2014; Miller, White, \& Boots, 2006).

In addition, phenotypic plasticity in host defences and parasite counter-defences may be of selective advantage depending on the level of parasite virulence and host defences and, therefore, may influence coevolutionary relationships between the host and parasite (Garland \& Kelly, 2006; Mougi, Kishida, \& Iwasa, 2011). Cooccurrence of inducible offenses and defences (i.e. reciprocal phenotypic plasticity) is well documented in antagonistic coevolutionary systems, and has been suggested as enhancing the evolutionary potential of species (Agrawal, 2001). For instance, plants induce defences that are dependent on the density of herbivores, and herbivores induce counter-defences that are dependent on the concentration of defences in consumed plants (Agrawal \& Karban, 2000). These antagonistic interactions may affect the probability of changes occurring in the host and, thus, of host and parasite diversification (Agrawal, 2001). Thus, parasite virulence, the relative importance of defensive tolerance of hosts and phenotypic plasticity of parasites and hosts may be related to each other and affect the evolution of hosts and parasites.

A particular type of parasitism is that in which individuals exploit the parental care of nonrelatives, thereby reducing the costs of parenting (parental-care parasitism; Roldán \& Soler, 2011). Obligate avian brood parasitism is an extreme form of parental-care parasitism, and an appropriate study system to test predictions related to a variety of coevolutionary scenarios and outcomes (Medina \& Langmore, 2016b), including the influence of coevolution in promoting species richness (Krüger, Sorenson, \& Davies, 2009) or the evolutionary rate of change of morphological traits of brood parasites (Medina \& Langmore, 2015). Here we suggest that virulence of the parasite and defensive strategies of the hosts, together with phenotypic plasticity in host defences and parasite counter-defences, would affect rates of specialization and speciation by brood parasites (see Fig. 1). We argue that less virulent brood-parasitic species (i.e. those that do not evict host eggs or nestlings) would differentially facilitate the evolution of defensive tolerance in their hosts, resulting in reduced rates of evolutionary change (i.e. specializations and speciation processes). Phenotypic plasticity of virulence of the parasites and of host defences would play a central role in this scenario since it affects specialization and speciation processes and its evolution would depend on particularities of host-parasite systems (see above). To exemplify these

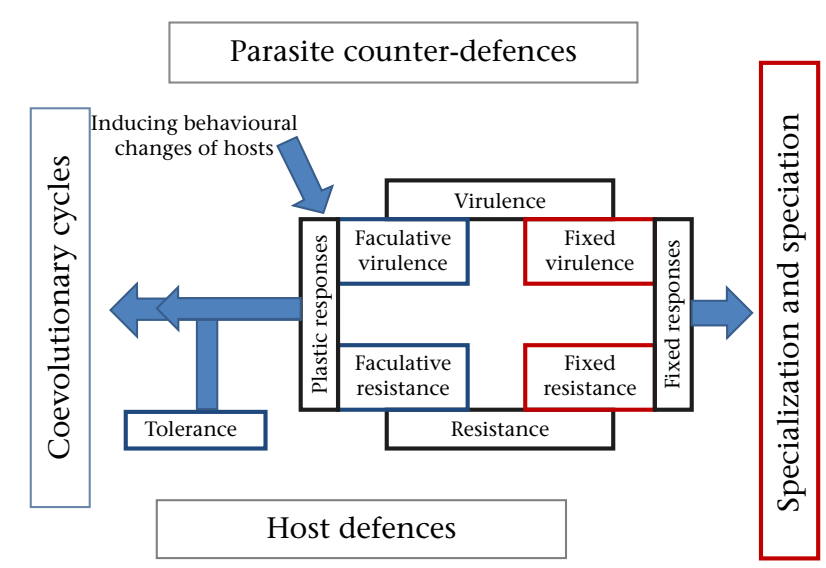

Figure 1. Relationships between characteristics of host defences and parasite counterdefences explaining parasite specialization and speciation on the one hand and coevolutionary cycles on the other. Special attention is paid to virulence of parasites and resistance and tolerance of hosts, which may be fixed or plastic responses to the parasite. Characteristics of hosts and parasites within the same colour frame indicate positive associations, while those of different colour frames are negatively related. points, we use particularities of evolutionary radiations and associated characteristics of brood parasites, mainly within the Family Cuculidae.

\section{PARASITE VIRULENCE AND THE EVOLUTIONARY RADIATION OF CUCKOOS}

Brood parasitism had a polyphyletic origin within the Family Cuculidae (Aragón, Møller, Soler, \& Soler, 1999; Sorenson \& Payne, 2002, 2005; but see ; Hughes, 2000). The Family includes 59 species of obligate brood parasites and 82 species with parental care distributed across all continents except Antarctica (Payne, 2005). The Cuculinae is the largest subfamily and includes two Tribes: the Cuculinii and the Phaenicophaeni. The former Tribe includes 11 genera and 51 species, all obligate brood parasites. Most of the species in the Phaenicophaeni are nesting cuckoos, but there are also four species of the brood-parasitic genus Clamator (Sorenson \& Payne, 2005). Interestingly, all species within the Cuculinii evict host chicks or kill them, while brood parasites of the genus Clamator are less virulent and sometimes allow some host chicks to survive together with the cuckoo chicks (Krüger et al., 2009). Thus, it is likely that, together with other particularities, differences in virulence of brood parasites explain the differential diversification experienced within each lineage. Notably, more than 50 years ago Friedmann (1964, page 95) wrote: 'The fact that Clamator, during its very long existence, has produced only 4 species, as against 12 in the younger Cuculus, or 12 in Chrysococcyx, coupled with the evolutionarily inert nature of its polymorphic trends, suggests that the genus is one that has been relatively less affected by evolutionary change'. Cuculus and Crysococcyx, within the Cuculinii, both include more species than the genus Clamator. Our point is that differences in virulence would select for different kinds of defences in hosts, and both affect the evolutionary process within different clades.

The great spotted cuckoo, Clamator glandarius, and the common cuckoo, Cuculus canorus, are the best known species of these two brood parasitic lineages, and may serve to exemplify differences between the lineages potentially responsible for the diversification of each group. The common cuckoo lays eggs that frequently mimic those of their hosts and, soon after hatching, their chicks evict the host eggs or nestlings (Davies, 2000). In contrast, the great spotted cuckoo lacks these adaptations (Soler \& Soler, 2000; Soler, Avilés, Soler, \& Møller, 2003). Killing host offspring is the most virulent behaviour of brood parasites. The common cuckoo nestlings evict all host eggs (or nestlings) in the nests soon after hatching (Honza, Voslajerova, \& Moskat, 2007), while those of the great spotted cuckoo outcompete host nestlings, although some sometimes survive. In addition, great spotted cuckoos are facultatively virulent since they are able to depredate host nests as a response to defensive resistance (egg ejection) of their hosts (Soler, Soler, Martínez, \& Møller, 1995). Thus, although both common cuckoos and great spotted cuckoos are able to kill all host offspring, the latter do so facultatively and the former obligately. The key point is that the reproductive success of hosts of nonevicting brood parasites is not fixed, and on average is higher than zero. These and some other differences between great spotted cuckoos and common cuckoos are useful for explaining the relatively higher radiation rate experienced by the Cuculinii along their evolutionary history.

A long-standing hypothesis in ecology is that specialization can lead to the generation of new species (Futuyma \& Moreno, 1988). Positive associations between specialization and speciation have been suggested for brood parasites (Davies, 2000; Krüger et al., 2009; but see ; Lanyon, 1992; Medina \& Langmore, 2016b) and, thus, factors affecting the former are central for explaining the 
latter. Body size (Krüger \& Davies, 2004), virulence (Kilner, 2005) and geographical distribution of parasites (Davies, 2000; Medina \& Langmore, 2016b) or hosts (Soler, Martín-Vivaldi, \& Møller, 2009) have all been suggested to affect specialization of brood parasites.

\section{Parasite-Induced Alteration of Host Behaviour}

Host behaviour and parasitism are inextricably linked (Ezenwa et al., 2016). Host behaviour frequently changes when hosts are infected with parasites, and this alteration of behaviour can occur for several reasons. For example, some host behavioural changes appear as a defence against parasites (Hart, 2011; Luong, Polak, \& Wedell, 2007) and others because of immunological or pathological consequences of parasite infection (Moore, 2002; Poulin, 1995). Some parasites are even able to manipulate host behaviour for their own benefit (Moore, 2002; Poulin, 2010). They can manipulate the phenotype of their hosts by either inducing changes in the host's appearance or taking control of host behaviour in ways that improve their probability of transmission or survival. This kind of manipulation has been documented in a few hundred distinct host-parasite associations corresponding to a large number of taxa, and is considered to be an adaptation of the parasite allowing them to enhance their own fitness (Moore, 2002; Poulin, 2007).

Some brood-parasitic species are also able to adaptively induce behavioural changes in their hosts. Some of these parasites' capabilities imply relatively low direct negative effects on host fitness and, for instance, include the 'distraction strategy' of great spotted cuckoos consisting of males flying very close to a nest of their magpie, Pica pica, hosts in which the female is incubating, while calling loudly. In this way, they attract the attention of the magpie female, which sometimes leaves the nest and attacks the cuckoo. This 'distraction strategy' provides the great spotted cuckoo female, which had previously positioned herself close by, with easy access to the host nest (Alvarez \& Arias de Reyna, 1974; Soler, Martínez, \& Soler, 1999). Another example of manipulative behaviour of brood parasites, with a relatively greater negative effect for hosts, is the ability of nestlings to manipulate feeding decisions of host foster parents. Brood-parasitic nestlings may increase the feeding effort of foster parents by, for instance, adjusting their begging intensity according to the size of host nestlings or the within-brood competition of a given host (Rivers, 2007; Tuero, Gloag, \& Reboreda, 2016), by mimicking nestling (Langmore, Maurer, Adcock, \& Kilner, 2008) or fledgling (De Mársico, Gantchoff, \& Reboreda, 2012) host call structures, or by producing begging calls that are attractive for foster parents (Gloag \& Kacelnik, 2013), or that mimic the sound of a complete brood of the host (Davies, Kilner, \& Noble, 1998; Gloag \& Kacelnik, 2013).

Other manipulative behaviours of brood parasites have more severe direct negative effects on their hosts: these include mafia behaviour, which is considered the clearest example of parasiteinduced alteration of host behaviour (Lefèvre et al., 2008, 2009). This behaviour involves the brood parasites revisiting the parasitized nests and, if the parasitic egg or nestling has been rejected, preying upon the host offspring (Zahavi, 1979). This mafia hypothesis has been experimentally demonstrated in the great spotted cuckoo-magpie system (Soler et al., 1995) and in the brown-headed cowbird, Molothrus ater,-prothonotary warbler, Protonotaria citrea, system (Hoover \& Robinson, 2007). By using this strategy, retaliatory brood parasites benefit from destroying host offspring because this behaviour induces hosts to renest and subsequently accept the parasitic offspring (Hoover \& Robinson, 2007; Soler et al., 1995). The expected host response to cuckoo retaliation has also been tested experimentally with magpies that suffered experimental predation after rejecting parasitic model eggs. Egg ejector magpies during the first breeding attempt changed to accepting parasitism in replacement clutches more often than expected by chance (Soler, Sorci, Soler, \& Møller, 1999). Thus, by imposing extra fitness costs reducing the fecundity of noncollaborative hosts, these brood parasites induce collaborative behaviour in their hosts, which decreases the strength of selection for host defences and, therefore, for parasite counter-defences (i.e. specialization). Theoretical models have also concluded that retaliation is a mechanism for the parasite to evade specialization (Abou Chakra, Hilbe, \& Traulsen, 2014).

\section{Defensive Resistance and Tolerance in Hosts Against Parasites}

More than 100 years ago, plant biologists clearly differentiated between resistance (i.e. the ability to prevent infection or limit parasite reproduction) and tolerance (i.e. acceptance of parasitism while minimizing the harm caused by the parasite) as two different mechanisms involved in plant defences against herbivores or pathogens. However, the study of tolerance has until recently been neglected by animal biologists, who have usually concentrated on the study of resistance (Råberg, 2014; Råberg et al., 2009). Tolerance is now considered an important defensive mechanism in animal-parasite interactions (Ayres \& Schneider, 2008; Boots, 2008; Medzhitov et al., 2012) and it is broadly accepted that tolerance is as adaptive as resistance for hosts (Medina \& Langmore, 2016a; Medzhitov et al., 2012; Svensson \& Råberg, 2010).

Both resistance and tolerance are likely to be costly and may have equivalent short-term benefits for individual hosts (Roy \& Kirchner, 2000). They may be considered as complementary defensive strategies and, consequently, the evolution of one may be related to the evolution of the other (Ayres \& Schneider, 2008; Råberg, Sim, \& Read, 2007). From an ecological and evolutionary perspective, they may have different evolutionary outcomes because tolerance and resistance responses to parasitism imply very different selective pressures on parasites. Host resistance, but not host tolerance, selects for counter-adaptation in parasites (but see Little, Shuker, Colegrave, Day, \& Graham, 2010). Importantly, since a tolerance response would not reduce the reproductive success of parasites, which otherwise directly depend on host resources, a pure defensive strategy of tolerance in hosts would strongly select against virulence-related traits in parasites (Little et al., 2010; Sternberg, Li, Wang, Gowler, \& de Roode, 2013). Thus, from an ecological perspective, tolerance would result in a larger population of parasites (i.e. higher prevalence of infection in the host population; Boots, 2008; Miller et al., 2006) of intermediate or nil virulence (Little et al., 2010; Sternberg et al., 2013). Instead, since the evolutionary outcome of host-parasite systems would largely depend on the strength of selection pressures on parasites (i.e. host defensive resistance) and on negative effects of parasites on hosts (i.e. virulence), the evolution of tolerance responses in hosts would result in a decreased rate of phenotypic evolution in both hosts and parasites (Best et al., 2014). Thus, resistance, but not tolerance, would increase parasite specialization and, therefore, speciation processes, because only resistance would result in open-ended antagonistic coevolution (Boots, 2008; Rausher, 2001). However, the evolution of tolerance favouring a higher prevalence of parasites allows their persistence (Roy \& Kirchner, 2000). Consequently, the degree of variation in both resistance and tolerance is expected to influence host-parasite dynamics (Hayward et al., 2014), including the evolution and diversification of parasites and their hosts (Best et al., 2014).

Tolerance has rarely been studied in scenarios of brood parasitism (Medina \& Langmore, 2016a), but it has been demonstrated to occur in the great spotted cuckoo-magpie system given that geographical variation in tolerance of brood parasitism by magpies 
(i.e. the slope of the relationship between breeding success of magpies and intensity of parasitism) covaries positively with the geographical variation in prevalence of parasitism by great spotted cuckoos (Soler et al., 2011). Thus, given the evolutionary scenario promoted by host resistance discussed above, and the likely spread of responses of tolerance by hosts that would buffer parasite evolution (Medina \& Langmore, 2016a), we suggest that the speed of evolutionary change in brood parasites and, therefore, their diversity, should be affected by the relative importance of the types of defence (tolerance or resistance) used by hosts. Host defences of resistance and of tolerance would trigger different evolutionary outcomes in parasite-host systems (Medina \& Langmore, 2016a; Roy \& Kirchner, 2000).

\section{Phenotypic Plasticity of Parasite Virulence and Host Resistance}

Above, we highlighted the importance of tolerance and virulence in determining the evolutionary processes involving hosts and parasites, scenarios in which phenotypic plasticity may play a preponderant role. This is because host responses of tolerance and resistance, as well as virulence of parasites, can be expressed plastically.

Phenotypic plasticity is a mechanism that allows animals to adjust their behaviour in response to changes in environmental conditions (Piersma \& van Gils, 2011; Via et al., 1995; WestEberhard, 2003). Given that host defences are costly (Sorci, Boulinier, Gauthier-Clerc, \& Faivre, 2009), it would be adaptive for hosts to be able to determine the risk of parasitism and plastically decrease host defences when the risk of parasitism declines (Michalakis, 2009). Phenotypic plasticity, however, is costly given that it needs to maintain the sensory and regulatory mechanisms required for acquiring information about the environment (i.e. risk of parasitism; DeWitt, Sih, \& Wilson, 1998; Ghalambor, McKay, Carroll, \& Reznick, 2007). Thus, phenotypic plasticity would be adaptive only when the risk of parasitism is highly variable and unpredictable.

The evolution of relaxed fixed host defences as a response to a predictable reduced risk of parasitism will allow the parasite to parasitize this species (or population) again (Dybdahl \& Lively, 1998; Nuismer, Doebeli, \& Browning, 2005) giving rise to coevolutionary cycles (Nuismer \& Thompson, 2006). The occurrence of cycles is based on frequency-dependent selection favouring rare genotypes in host populations (Bell, 1982; Dybdahl \& Lively, 1998; Nuismer et al., 2005) and/or in parasites choosing the host species or population with the least evolved defences (Davies \& Brooke, 1989; Martín-Gálvez et al., 2007; Nuismer \& Thompson, 2006).

Theoretically, the general ideas discussed above should also be valid for brood parasite-host systems. Coevolutionary cycles based on fluctuating selection, as well as plastic responses by hosts, should be frequent outcomes in antagonistic coevolutionary interactions. However, this does not seem to be the case in many brood parasite-host systems in which brood parasites have stopped parasitizing many host species that have retained the ability to recognize and eject parasitic eggs (successful defence; Soler, 2014). Moreover, in his review of the characteristics of brood parasitism in cuckoos and cowbirds, Soler (2014) reported evidence of coevolutionary cycles for only five host species of the common cuckoo plus one host species of the shiny cowbird, Molothrus bonariensis. Interestingly, all these species show phenotypic plastic responses in relation to risk of parasitism (i.e. egg rejection of parasitic eggs). In addition, as well as having coevolutionary cycles and plastic responses of resistance, their rates of parasitic egg rejection are intermediate (Soler, 2014). Thus, intermediate and plastic responses of resistance by hosts are apparently linked to the occurrence of coevolutionary cycles in brood parasite-host systems, which would consequently affect the specialization and speciation of parasites.

Conditional plastic responses to experimental parasitism have been reported in only three current host species of the common cuckoo, all with an intermediate rejection rate (reed warbler, Acrocephalus scirpaceus: Barabás, Gilicze, Takasu, \& Moskát, 2004; Brooke, Davies, \& Noble, 1998; Stokke et al., 2008; rufous-tailed scrub robin, Cercotrichas galactotes: Alvarez, 1996; Soler, MartínVivaldi, \& Fernández-Morante, 2012; meadow pipit, Anthus pratensis: Moksnes \& Røskaft, 1989). At least the first two species are also involved in coevolutionary cycles with the common cuckoo (Soler, 2014). Other reports cited in Soler (2014) suggest that fixed responses are frequent in hosts of the common cuckoo. Of the 49 host species, 12 eject $100 \%$ and three $0 \%$ of experimental parasitic eggs. These 15 species are not currently used as hosts except for the common chiffchaff, Phylloscopus collybita, the Lapland longspur, Calcarius lapponicus, and the common stonechat, Saxicola torquatus, which are rarely used (Soler, 2014). Thus, because none of these species are involved in coevolutionary cycles (i.e. defences are not relaxed in periods of no parasitism), their responses of resistance to experimental parasitism can be considered fixed and nonplastic. Moreover, given the apparently positive association between plastic responses and coevolutionary cycles, it can be concluded that fixed responses and the absence of coevolutionary cycles are relatively common in this host-brood parasite system. On the other side of the coin, the most apparent virulent behaviour of the common cuckoo, i.e. eviction behaviour by parasitic hatchlings, is genetically fixed and invariably expressed (nonphenotypically plastic behaviour; Davies, 2000). Thus, it is possible that the fixed high virulence of cuckoos is responsible for the relatively frequent nonplastic defences of their hosts.

However, this association is hardly comparable to other systems since a similar percentage of hosts of the less virulent brownheaded cowbird listed in Soler (2014) show fixed responses (21 of the 62 host species eject 100\% of experimental eggs and are currently not used as hosts, but the eight corvids in the list could once have been hosts of a now extinct brood parasite; Soler, 2016). Brood-parasitic cowbirds (Genus Molothrus) include only five species, two of them specialist parasites of other icterids. Thus, the similar frequency of fixed responses of hosts of brown-headed cowbirds and common cuckoos does not result in a similar rate of diversification of the brood-parasitic lineages to which they belong. Consequently, it is likely that the expression of nonplastic virulent characters by the common cuckoo, and not fixed responses of their hosts, was the characteristic that has played a major role in the specialization of the Cuculinii tribe.

The two main hosts of the great spotted cuckoo in Europe, the magpie and the carrion crow, Corvus corone, show intermediate and no resistance defences, respectively. Evidence of cycling coevolution exists for magpies (Martín-Gálvez et al., 2007; Soler, Soler, Martínez, Pérez-Contreras, \& Møller, 1998) in association with enormously variable egg ejection rates. Moreover, egg ejection varies depending on population parasitism rates (Soler, Martínez, Soler, \& Møller, 1999, 2001; Soler, Martin-Galvez, De Neve, \& Soler, 2013; Soler, Perez-Contreras, \& Soler, 2015; Soler, Soler, et al., 1998; Soler, Sorci, et al., 1999) suggesting phenotypic plasticity, and this has been experimentally demonstrated. Magpies that were experimentally parasitized and rejected model eggs changed their response and accepted parasitic eggs in their replacement clutches after suffering nest predation (Soler, Sorci, et al., 1999). Parasitism by great spotted cuckoos is enormously variable between years and/or populations in both of its main host species, the magpie and the carrion crow (Baglione et al., 2017; Soler, 1990; Soler, Soler, et al., 1998; Soler, Soler, Pérez-Contreras, \& Martínez, 2002). Thus, in line with the theoretical framework discussed above, the 
uncertainty of a parasitism selection pressure should explain the evolution of plastic defensive responses in magpies. On the other side of the coevolutionary interaction, we also know that great spotted cuckoos are more virulent against hosts that express resistance defences (i.e. rejection of parasitic eggs; see above). Consequently, the plastic virulence of the brood parasite may also play a role in determining the evolution of plastic responses in their hosts. This should invariably be the case for the magpie-great spotted cuckoo system where virulence is expressed facultatively by the parasite towards hosts that show defensive resistance (Soler, Møller, \& Soler, 1998).

Interestingly, plastic virulence (i.e. mafia behaviour) is also known for brown-headed cowbirds. However, information on plasticity of host responses is in general scarce, and has been detected for a single host of the shiny cowbird (Hoover \& Robinson, 2007). Evidence of strategic destruction of host eggs has been found for this species (Fiorini, Gloag, Kacelnik, \& Reboreda, 2014) and, thus, it is a possible system for investigating facultative virulence further. More experimental studies are therefore necessary to confirm an association between plastic virulence of parasites and plastic responses of hosts in this group of brood parasites. In any case, in comparison with brood parasites of the Tribe Cuculinii, the genera Molothrus and Clamator show relatively lower virulence which, at least in some species, can be expressed facultatively. Moreover, both genera include a relatively low number of species (i.e. lower diversification), which tentatively supports the proposed relationship between fixed virulence and diversification of brood parasite lineages.

\section{GENERAL DISCUSSION}

Above, we have argued that (1) parasite virulence, (2) ability of parasites to manipulate host behaviour, (3) relative importance of host defensive tolerance and resistance or (4) plasticity in host defensive traits and in parasite virulence would affect the coevolutionary process between hosts and parasites and, therefore, evolutionary change of parasites. Here, we explore how all these factors can be related to each other and together explain variation in rates of diversification of parasites (Fig. 1). Some parasites can influence host plastic responses to parasitism, which, in addition to virulence, may contribute to explaining parasite evolution. We know that some retaliatory brood parasites can induce changes in defensive responses of their hosts and force them to accept parasitism (Hoover \& Robinson, 2007; Soler et al., 1995). In this case, hosts would be able to defend against parasites mainly by showing tolerance. For parasites, this would result in a low level of selection pressure and, thus, higher evolutionary stability (i.e. low rate of evolutionary change; Abou Chakra et al., 2014). Interestingly, this scenario where parasites alter host defensive behaviour requires parasites to be facultatively virulent and resistance defences of hosts to be expressed plastically (Soler, Møller, et al., 1998). Even if highly virulent brood parasites do induce hosts to renest, host acceptance of parasitism would only be selected over resistance if parasites show reduced virulence (i.e. hosts have a chance to rear some of their own offspring) as a response to host compliance. Thus, not only parasite virulence but also the ability to alter host defensive behaviour, together with plastic responses of hosts (plastic resistance) and parasites (facultative virulence), should be important factors explaining rates of speciation of brood parasites and of hosts. Below, we try to answer questions related to differences between the coevolutionary systems of the common cuckoo and great spotted cuckoo to further explain the importance of such parasite and host characteristics in the coevolutionary process and outcomes.
(1) Why is phenotypic plasticity more frequent in great spotted cuckoo hosts than in common cuckoo hosts? Probably, this is related to the parasitism selection pressure by the great spotted cuckoo being less predictable than that of the common cuckoo, in terms of either rates of parasitism or virulence of the parasites. In addition, the great spotted cuckoo induces behavioural changes in magpies by behaving more virulently against hosts that show resistance responses. The probability of retaliation is likely to depend on the parasitism selection pressure (i.e. parasite prevalence), and therefore may also be largely unpredictable. Thus, the unpredictability of both parasitism and cuckoo retaliation will select for plastic defensive traits of resistance in magpies.

(2) Why has defensive tolerance been detected in hosts of the great spotted cuckoos but not in those of common cuckoos? First, the great spotted cuckoo is the only brood parasite species in which this has been studied. However, it is also true that characteristics of the great spotted cuckoo-magpie system, but not those of the common cuckoo, predict the evolution of defensive tolerance (Svensson \& Råberg, 2010). Great spotted cuckoos can induce magpies that show resistance to change their behaviour and tolerate parasitism. Moreover, great spotted cuckoos, but not common cuckoos, show facultative virulence against their hosts which is related to the latter's phenotypic plastic resistance and, thus, would facilitate the evolution of defensive tolerance in their hosts (Fig. 1). Fixed virulence seems to be the rule for the common cuckoo-host system and, thus, a reduced opportunity for defensive tolerance exists for hosts in this system (Fig. 1).

(3) Why is the genus Cuculus (i.e. Tribe Cuculinii) more diverse than the genus Clamator? We speculate that it is because brood parasites of the genus Cuculus are characterized by fixed and high virulent behaviour (i.e. eviction behaviour of parasitic hatchlings; Honza et al., 2007), and their hosts mainly show resistance (Soler, 2014). On average, species of the genus Clamator show relatively low levels of virulence (i.e. no eviction behaviour of their hatchlings) and, at least for great spotted cuckoos, certain virulent behaviours are facultatively expressed. Facultative virulence favours defensive tolerance in their hosts, and all these characteristics favour coevolutionary cycles, reducing processes of specialization, and therefore of speciation (Fig. 1). Thus, the fixed high virulence of cuckoos that reduce the probability of defensive tolerance evolving seems to be responsible for the low evolutionary change in the genus Clamator emphasized by Friedmann (1964) half a century ago.

\section{Conclusions and prospects}

Here, we propose that high virulence of parasites, defensive resistance of hosts and fixed responses of hosts and parasites lead to the arms race between brood parasites and hosts escalating much more rapidly, which considerably increases the speed of evolutionary specialization and the probability of speciation events. Coevolutionary cycles with low opportunities of parasite specialization and speciation are likely to occur in brood parasites with relatively low virulence, defensive tolerance of hosts and plastic responses of hosts and parasites. Thus, when studying the diversification of brood parasites within the avian phylogeny, considering these three factors would help us to understand the reasons for their evolution. Here we have illustrated these points by considering egg ejection and egg acceptance as defensive resistance and tolerance of hosts, and virulence as the key counter-defence of brood parasites. The same reasoning would, however, apply to other lines of host defence. Moreover, we have not considered alternative explanations for the differences in species richness we have used to exemplify our points. However, when testing these differences in the geographical ranges of the group considered or 
any other character known to affect specialization and speciation, these alternative explanations should be considered.

Particularly interesting for future studies would be to explore the effect of facultative virulence of brood parasites and tolerance of their hosts on speciation rates of different parasite lineages. We already know that virulence is associated with parasite specialization (Medina \& Langmore, 2016b). Further attempts to detect evidence of defensive tolerance in hosts and of facultative virulence in parasites are necessary to test the expected negative association with specialization and speciation.

Future studies should also concentrate on exploring and detecting defensive tolerance in different brood parasite-host systems, which should be more commonly detected in hosts of parasites that show more virulence against resistant hosts. The importance of phenotypic plasticity in the evolution of interactions is well recognized (Agrawal, 2001), but little is known of its role in the evolution of defensive tolerance. We also predict that defensive tolerance should be more frequently detected in hosts of brood parasites belonging to parasite lineages that show relatively little diversification.

We hope that the ideas and predictions described here will encourage further research to try to understand the importance of tolerance, plasticity and virulence for the evolution of parasite-host relationships in general and the diversification of brood parasites in particular.

\section{Acknowledgments}

We thank two anonymous referees for constructive comments on the manuscript, which considerably improved and clarified the text. While writing this essay, we benefited from funding by the Spanish Ministerio de Ciencia e Innovación, Ministerio de Economía y Competitividad and European funds (FEDER) (project CGL201348193-C3-1-P) to J.J.S., and the Junta de Andalucía (project RNM610) to M.S.

\section{References}

Abou Chakra, M., Hilbe, C., \& Traulsen, A. (2014). Plastic behaviors in hosts promote the emergence of retaliatory parasites. Scientific Reports, 4, 4251. http:// dx.doi.org/10.1038/srep04251.

Agrawal, A. A. (2001). Phenotypic plasticity in the interactions and evolution of species. Science, 294, 321-326. http://dx.doi.org/10.1126/science.1060701.

Agrawal, A. A., \& Karban, R. (2000). Specificity of constitutive and induced resistance: Pigment glands influence mites and caterpillars on cotton plants. Entomologia Experimentalis et Applicata, 96, 39-49. http://dx.doi.org/10.1046/j.15707458.2000.00677.x.

Alvarez, F. (1996). Model cuckoo Cuculus canorus eggs accepted by Rufous bush chats Cercotrichas galactotes during the parasite's absence from the breeding area. Ibis, 138, 340-342.

Alvarez, F., \& Arias de Reyna, L. (1974). Mecanismos de parasitiación por Clamator glandarius y defensa por Pica pica. Doñana, Acta Vertebrata, 1, 43-65.

Aragón, S., Møller, A. P., Soler, J. J., \& Soler, M. (1999). Molecular phylogeny of cuckoos supports a polyphyletic origin of brood parasitism. Journal of Evolutionary Biology, 12, 495-506.

Ayres, J. S., \& Schneider, D. S. (2008). A signaling protease required for melanization in Drosophila affects resistance and tolerance of infections. PLoS Biology, 6, 305

Baglione, V., Bolopo, D., Canestrari, D., Martínez, J. G., Roldan, M., Vila, M., et al. (2017). Spatiotemporal variation of host use in a brood parasite: The role of the environment. Behavioral Ecology, 28(1), 49-58.

Barabás, L., Gilicze, B., Takasu, F., \& Moskát, C. (2004). Survival and anti-parasite defense in a host metapopulation under heavy brood parasitism: A source-sink dynamic model. Journal of Ethology, 22, 143-151.

Bell, G. (1982). The masterpiece of nature: The evolution and genetic of sexuality. Berkeley, CA: University of California Press.

Best, A., White, A., \& Boots, M. (2014). The coevolutionary implications of host tolerance. Evolution, 68, 1426-1435.

Boots, M. (2008). Fight or learn to live with the consequences? Trends in Ecology and Evolution, 23, 248-250. http://dx.doi.org/10.1016/j.tree.2008.01.006.

Brooke, M. D. L., Davies, N. B., \& Noble, D. G. (1998). Rapid decline of host defenses in response to reduced cuckoo parasitism: Behavioral flexibility of reed warblers in a changing world. Proceedings of the Royal Society B: Biological Sciences, $265,1277-1282$

Davies, N. B. (2000). Cuckoos, cowbirds, and others cheats. London, U.K.: T \& A D Poyser.
Davies, N. B., \& Brooke, M.d. L. (1989). An experimental study of coevolution between the cuckoo, Cuculus canorus, and its host. II. Host egg markings, chick discrimination and general discussion. Journal of Animal Ecology, 58, 225-236.

Davies, N. B., Kilner, R. M., \& Noble, D. G. (1998). Nestling cuckoos, Cuculus canorus, exploit hosts with begging calls that mimic a brood. Proceedings of the Royal Society B: Biological Sciences, 265, 673-678.

Dawkins, D., \& Krebs, J. R. (1979). Arms races between and within species. Proceedings of the Royal Society B: Biological Sciences, 205, 489-511.

De Mársico, M. C., Gantchoff, M. G., \& Reboreda, J. C. (2012). Host-parasite coevolution beyond the nestling stage? Mimicry of host fledglings by the specialist screaming cowbird. Proceedings of the Royal Society B: Biological Sciences, 279, 3401-3408.

DeWitt, T. J., Sih, A., \& Wilson, D. S. (1998). Costs and limits of phenotypic plasticity Trends in Ecology and Evolution, 13, 77-81.

Dybdahl, M. F., \& Lively, C. M. (1998). Host-parasite coevolution: Evidence for rare advantage and time-lagged selection in a natural population. Evolution, 52, 1057-1066.

Ezenwa, V. O., Archie, E. A., Craft, M. E., Hawley, D. M., Martin, L. B., Moore, J., et al. (2016). Host behaviour-parasite feedback: An essential link between animal behaviour and disease ecology. Proceedings of the Royal Society B: Biological Sciences, 283. http://dx.doi.org/10.1098/rspb.2015.3078.

Fiorini, V. D., Gloag, R., Kacelnik, A., \& Reboreda, J. C. (2014). Strategic egg destruction by brood-parasitic cowbirds? Animal Behaviour, 93, 229-235.

Friedmann, H. (1964). Evolutionary trends in the avian genus Clamator. Smithsonian Miscellaneous Collection, 146, 1-127.

Futuyma, D. J., \& Moreno, G. (1988). The evolution of ecological specialization. Annual Review of Ecology and Systematics, 19, 207-233.

Garland, T., Jr., \& Kelly, S. A. (2006). Phenotypic plasticity and experimental evolution. Journal of Experimental Biology, 209, 2344-2361.

Ghalambor, C. K., McKay, J. K., Carroll, S. P., \& Reznick, D. N. (2007). Adaptive versus non-adaptive phenotypic plasticity and the potential for contemporary adaptation in new environments. Functional Ecology, 21, 394-407.

Gloag, R., \& Kacelnik, A. (2013). Host manipulation via begging call structure in the brood-parasitic shiny cowbird. Animal Behaviour, 86, 101-109.

Hart, B. L. (2011). Behavioural defences in animals against pathogens and parasites: Parallels with the pillars of medicine in humans. Philosophical Transactions of the Royal Society of London B: Biological Sciences, 366, 3406. http://dx.doi.org/ 10.1098/rstb.2011.0092.

Hayward, A. D., Nussey, D. H., Wilson, A. J., Berenos, C., Pilkington, J. G., Watt, K. A. et al. (2014). Natural selection on individual variation in tolerance of gastrointestinal nematode infection. PLoS Biology, 12, e1001917. http://dx.doi.org/ 10.1371/journal.pbio.1001917.

Honza, M., Voslajerova, K., \& Moskat, C. (2007). Eviction behaviour of the common cuckoo Cuculus canorus chicks. Journal of Avian Biology, 38, 385-389.

Hoover, J. P., \& Robinson, S. K. (2007). Retaliatory mafia behavior by a parasitic cowbird favors host acceptance of parasitic eggs. Proceedings of the National Academy of Sciences of the Uinted States of America, 104, 4479-4483.

Hughes, J. M. (2000). Monophyly and phylogeny of cuckoos (Aves, Cuculidae) inferred from osteological characters. Zoological Journal of the Linnean Society 130, 263-307.

Kilner, R. M. (2005). The evolution of virulence in brood parasites. Ornithological Science, 4, 55-64.

Krüger, O., \& Davies, N. B. (2004). The evolution of egg size in the brood parasitic cuckoos. Behavioral Ecology, 15, 210-218.

Krüger, O., Sorenson, M. D., \& Davies, N. B. (2009). Does coevolution promote species richness in parasitic cuckoos? Proceedings of the Royal Society B: Biological Sciences, 276, 3871-3879. http://dx.doi.org/10.1098/rspb.2009.1142.

Langmore, N., Maurer, G., Adcock, G. J., \& Kilner, R. M. (2008). Socially acquired hostspecific mimicry and the evolution of host races in Horsfield's bronze-cuckoo Chalcites basalis. Evolution, 62, 1689-1699.

Lanyon, S. M. (1992). Interspecific brood parasitism in blackbirds (Icterinae): A phylogenetic perspective. Science, 225, 77-79.

Lefèvre, T., Adamo, S. A., Biron, D. G., Missé, D., Hughes, D., \& Thomas, F. (2009) Invasion of the body snatchers: The diversity and evolution of manipulative strategies in host-parasite interactions. Advances in Parasitology, 68, 45-83.

Lefèvre, T., Roche, B., Poulin, R., Hurd, H., Renaud, F., \& Thomas, F. (2008). Exploiting host compensatory responses: The 'must' of manipulation? Trends in Parasitology, 24, 435-439. http://dx.doi.org/10.1016/j.pt.2008.06.006.

Little, T. J., Shuker, D. M., Colegrave, N., Day, T., \& Graham, A. L. (2010). The coevolution of virulence: Tolerance in perspective. PLoS Pathogens, 6, e1001006. http://dx.doi.org/10.1371/journal.ppat.1001006.

Luong, L. T., Polak, M., \& Wedell, N. (2007). Costs of resistance in the Drosophilamacrocheles system: A negative genetic correlation between ectoparasite resistance and reproduction. Evolution, 61,1391-1402. http://dx.doi.org/10.1111/ j.1558-5646.2007.00116.x.

Martín-Gálvez, D., Soler, J. J., Martínez, J. G., Krupa, A. P., Soler, M., \& Burke, T. (2007). Cuckoo parasitism and productivity in different magpie subpopulations predict frequencies of the 457bp allele: A mosaic of coevolution at a small geographic scale. Evolution, 61, 2340-2348. http://dx.doi.org/10.1111/j.1558-5646.2007.00194.x.

Medina, I., \& Langmore, N. E. (2015). Coevolution is linked with phenotypic diversification but not speciation in avian brood parasites. Proceedings of the Royal Society B: Biological Sciences, 282. http://dx.doi.org/10.1098/rspb.2015.2056.

Medina, I., \& Langmore, N. E. (2016a). The evolution of acceptance and tolerance in hosts of avian brood parasites. Biological Reviews, 91, 569-577. 
Medina, I., \& Langmore, N. E. (2016). The evolution of host specialisation in avian brood parasites. Ecology Letters, 19, 1110-1118.

Medzhitov, R., Schneider, D. S., \& Soares, M. P. (2012). Disease tolerance as a defense strategy. Science, 335, 936-941.

Michalakis, Y. (2009). Parasitism and the evolution of life-history traits. In F. Thomas, J. F. Guégan, \& F. Renaud (Eds.), Ecology and evolution of parasitim (pp. 19-30). Oxford, U.K.: Oxford University Press.

Miller, M. R., White, A., \& Boots, M. (2006). The evolution of parasites in response to tolerance in their hosts: The good, the bad, and apparent commensalism. Evolution, 60, 945-956.

Moksnes, A., \& Røskaft, E. (1989). Adaptations of meadow pipits to parasitism by the common cuckoo. Behavioral Ecology and Sociobiology, 24, 25-30.

Moore, J. (2002). Parasites and the behavior of animals. Oxford, U.K.: Oxford University Press.

Mougi, A., Kishida, O., \& Iwasa, Y. (2011). Coevolution of phenotypic plasticity in predator and prey: Why are inducible offenses rarer than inducicle defenses? Evolution, 65, 1079-1087. http://dx.doi.org/10.1111/j.1558-5646.2010.01187.x.

Nuismer, S. L., Doebeli, M., \& Browning, D. (2005). The coevolutionary dynamics of antagonistic interactions mediated by quantitative traits with evolving variances. Evolution, 59, 2073-2082.

Nuismer, S. L., \& Thompson, J. N. (2006). Coevolutionary alteration in antagonistic interactions. Evolution, 60, 2207-2217.

Payne, R. B. (2005). The cuckoos. New York, NY: Oxford University Press.

Piersma, T., \& van Gils, J. A. (2011). The flexible phenotype: A body-centred integration of ecology, physiology and behaviour. Oxford, U.K.: Oxford University Press.

Poulin, R. (1995). 'Adaptive' changes in the behaviour of parasitized animals: A critical review. International Journal for Parasitology, 25, 1371-1383.

Poulin, R. (2007). Evolutionary ecology of parasites. Princeton, NJ: Princeton University Press.

Poulin, R. (2010). Parasite manipulation of host behavior: An update and frequently asked questions. In H. J. Brockmann (Ed.), Advances in the Study of Behavior (Vol. 41, pp. 151-186). London, U.K.: Academic Press.

Råberg, L. (2014). How to live with the enemy: Understanding tolerance to parasites. PLoS Biology, 12, e1001989. http://dx.doi.org/10.1371/journal pbio.1001989.

Råberg, L., Graham, A. L., \& Read, A. F. (2009). Decomposing health: Tolerance and resistance to parasites in animals. Philosophical Transactions of the Royal Society of London B: Biological Sciences, 364, 37-49. http://dx.doi.org/10.1098/ rstb.2008.0184.

Råberg, L., Sim, D., \& Read, A. F. (2007). Disentangling genetic variation for resistance and tolerance to infectious diseases in animals. Science, 318, 812-814. http:/ dx.doi.org/10.1126/science.1148526.

Rausher, M. D. (2001). Co-evolution and plant resistance to natural enemies. Nature, $411,857-864$.

Rivers, J. W. (2007). Nest mate size, but not short-term need, influences begging behavior of a generalist brood parasite. Behavioral Ecology, 18, 222-230.

Roldán, M., \& Soler, M. (2011). Parental-care parasitism: How do unrelated offspring attain acceptance by foster parents? Behavioral Ecology, 22, 679-691.

Roy, B. A., \& Kirchner, J. W. (2000). Evolutionary dynamics of pathogen resistance and tolerance. Evolution, 54, 51-63.

Schmid-Hempel, P. (2011). Evolutionary parasitology. The integrated study of infections, immunology, ecology, and genetics. Oxford, U.K.: Oxford University Press.

Soler, M. (1990). Relationships between the great spotted cuckoo Clamator glandarius and its corvid hosts in a recently colony area. Ornis Scandinavica, 21, $212-223$.

Soler, M. (2014). Long-term coevolution between avian brood parasites and their hosts. Biological Reviews, 89, 688-704.

Soler, M. (2016). Brood parasite-host coevolution in America versus Europe: Egg rejection in large-sized host species. Ardeola, 63, 35-48. http://dx.doi.org 10.13157/arla.63.1.2016.rp2.

Soler, J. J., Avilés, J. M., Soler, M., \& Møller, A. P. (2003). Evolution of host egg mimicry in a brood parasite, the great spotted cuckoo. Biological Journal of the Linnean Society, 79, 551-563.

Soler, J. J., Martin-Galvez, D., De Neve, L., \& Soler, M. (2013). Brood parasitism correlates with the strength of spatial autocorrelation of life history and defensive traits in Magpies. Ecology, 94, 1338-1346.

Soler, J. J., Martín-Gálvez, D., Martínez, J. G., Soler, M., Canestrari, D., Abad Gómez, J. M., et al. (2011). Evolution of tolerance by magpies to brood parasitism by great spotted cuckoos. Proceedings of the Royal Society B: Biological Sciences, 278, 2047-2052. http://dx.doi.org/10.1098/rspb.2010.2218.

Soler, M., Martín-Vivaldi, M., \& Fernández-Morante, J. (2012). Conditional response by hosts to parasitic eggs: The extreme case of the rufous-tailed scrub robin. Animal Behaviour, 84, 421-426. http://dx.doi.org/10.1016/j.anbehav.2012.05.016.

Soler, J. J., Martín-Vivaldi, M., \& Møller, A. P. (2009). Geographic distribution of suitable hosts explains the evolution of specialized gentes in the European cuckoo Cuculus canorus. BMC Evolutionary Biology, 9, 88.

Soler, M., Martínez, J. G., \& Soler, J. J. (1999c). Nest defence by magpies (Pica pica) and the brood parasitic great spotted cuckoo (Clamator glandarius) in parasitized and unparasitized nests. Journal für Ornithology, 140, 199-205.

Soler, J. J., Martínez, J. G., Soler, M., \& Møller, A. P. (1999). Genetic and geographic variation in rejection behavior of cuckoo eggs by European magpie populations: An experimental test of rejecter-gene flow. Evolution, 53, 947-956.

Soler, J. J., Martínez, J. G., Soler, M., \& Møller, A. P. (2001). Coevolutionary interactions in a host-parasite system. Ecology Letters, 4, 470-476. http:/ dx.doi.org/10.1046/j.1461-0248.2001.00247.x.

Soler, J. J., Møller, A. P., \& Soler, M. (1998). Mafia behaviour and the evolution of facultative virulence. Journal of Theoretical Biology, 191, 267-277.

Soler, M., Perez-Contreras, T., \& Soler, J. J. (2015). Synchronization of laying by great spotted cuckoos and recognition ability of magpies. Journal of Avian Biology, 46, $608-615$.

Soler, J. J., \& Soler, M. (2000). Brood-parasite interactions between great spotted cuckoos and magpies: A model system for studying coevolutionary relationships. Oecologia, 125, 309-320.

Soler, M., Soler, J. J., Martínez, J. G., \& Møller, A. P. (1995). Magpie host manipulation by great spotted cuckoos: Evidences for an avian mafia? Evolution, 49, 770-775.

Soler, M., Soler, J. J., Martínez, J. G., Pérez-Contreras, T., \& Møller, A. P. (1998). Microevolutionary change and population dynamics of a brood parasite and its primary host: The intermittent arms race hypothesis. Oecologia, 117, 381-390.

Soler, M., Soler, J. J., Pérez-Contreras, T., \& Martínez, J. G. (2002). Differential reproductive success of great spotted cuckoos Clamator glandarius parasitising magpies Pica pica and carrion crows Corvus corone: The importance of parasitism costs and host defences. Avian Science, 2, 25-32.

Soler, J. J., Sorci, G., Soler, M., \& Møller, A. P. (1999). Change in host rejection behavior mediated by the predatory behavior of its brood parasite. Behavioral Ecology, 10, 275-280. http://dx.doi.org/10.1093/beheco/10.3.275.

Sorci, G., Boulinier, T., Gauthier-Clerc, M., \& Faivre, B. (2009). The evolutionary ecology of the immune response. In F. Thomas, J. F. Guégan, \& F. Renaud (Eds.), Ecology and evolution of parasitim (pp. 5-17). Oxford, U.K.: Oxford University Press.

Sorenson, M. D., \& Payne, R. B. (2002). Molecular genetic perspectives on avian brood parasitism. Integrative and Comparative Biology, 142, 388-400.

Sorenson, M. D., \& Payne, R. B. (2005). A molecular genetic analysis of cuckoo phylogeny. In R. B. Payne (Ed.), The cuckoos (pp. 68-94). Oxford, U.K.: Oxford University Press.

Sternberg, E. D., Li, H., Wang, R., Gowler, C., \& de Roode, J. C. (2013). Patterns of hostparasite adaptation in three populations of monarch butterflies infected with a naturally occurring protozoan disease: Virulence, resistance, and tolerance. American Naturalist, 182, E235-E248.

Stokke, B. G., Hafstad, I., Rudolfsen, G., Moksnes, A., Møller, A. P., Røskaft, E., et al. (2008). Predictors of resistance to brood parasitism within and among reed warbler populations. Behavioral Ecology, 19, 612-620.

Svensson, E. I., \& Råberg, L. (2010). Resistance and tolerance in animal enemy-victim coevolution. Trends in Ecology and Evolution, 25, 267-274. http://dx.doi.org/ 10.1016/j.tree.2009.12.005.

Thompson, J. N. (1994). The coevolutionary process. Chicago, IL: University of Chicago Press.

Tuero, D. T., Gloag, R., \& Reboreda, J. C. (2016). Nest environment modulates begging behavior of a generalist brood parasite. Behavioral Ecology, 27, 204-210.

Via, S., Gomulkiewicz, R., De Jong, G., Scheiner, S. M., Schlichting, C. D., \& Van Tienderen, P. H. (1995). Adaptive phenotypic plasticity: Consensus and controversy. Trends in Ecology and Evolution, 10, 212-217. http://dx.doi.org/10.1016/ S0169-5347(00)89061-8.

West-Eberhard, M. J. (2003). Developmental plasticity and evolution. New York, NY: Oxford University Press.

Zahavi, A. (1979). Parasitism and nest predation in parasitic cuckoos. American Naturalist, 113, 157-159. 